\title{
Detection of X-ray line emission from the shell of SNR B0540-69.3 with XMM-Newton RGS*
}

\author{
K. J. van der Heyden ${ }^{1}$, F. Paerels ${ }^{2}$, J. Cottam ${ }^{2}$, J. S. Kaastra ${ }^{1}$, and G. Branduardi-Raymont ${ }^{3}$ \\ 1 SRON Laboratory for Space Research, Sorbonnelaan 2, 3584 CA Utrecht, The Netherlands \\ 2 Columbia Astrophysics Laboratory, Columbia University, 538 West 120th Street, New York, NY 10027, USA \\ 3 Mullard Space Science Laboratory, University College London, Holmbury St. Mary, Dorking, \\ Surrey RH5 6NT, UK
}

Received 3 October 2000/ Accepted 6 November 2000

\begin{abstract}
We present X-ray observations of PSR 0540-69.3 with the XMM-Newton observatory. The spectra obtained with the Reflection Grating Spectrometer reveal, for the first time, emission from ionized species of O, $\mathrm{Ne}$ and Fe originating from the SNR shell. Analysis of the emission line spectrum allows us to derive estimates of the temperature, ionization timescale, abundances, location, and velocity of the emitting gas.
\end{abstract}

Key words. ISM: individual: SNR 0540-69.3 - ISM: supernova - X-rays: ISM

\section{Introduction}

SNR 0540-69.3 is one of only a few Crab-like supernova remnants (SNRs) with a shell. SNR0540-69.3 harbors a pulsar, PSR B0540-69.3, discovered in the soft X-ray band by Seward et al. (1984). Like the Crab, it shows plerion emission from a synchrotron nebula powered by the embedded young pulsar (Chanan et al. 1984). From the period $(50 \mathrm{~ms})$ and spin down rate $\left(4.7910^{-13} \mathrm{~s}^{-1}\right)$ a characteristic age of $1660 \mathrm{yr}$ and rotational energy loss rate of $1.510^{38} \mathrm{ergs} \mathrm{s}^{-1}$ have been derived (Seward et al. 1984).

Early optical observations (Mathewson et al. 1980) classified SNR 0540-69.3 as a young oxygen-rich SNR with an $8^{\prime \prime}$ diameter shell, bright in [O III]. Mathewson et al. (1980) also found a diffuse patch of $[\mathrm{O}$ III $] \sim 30^{\prime \prime}$ to the west of the ring which indicated that the remnant was larger than $8^{\prime \prime}$. A ROSAT High Resolution Imager observation by Seward \& Harnden (1994) also revealed emission from well outside the central region, which they interpreted as from a patchy outer shell with a diameter of $55^{\prime \prime}$. This shell contributes $\sim 20 \%$ to the measured flux in the ROSAT 0.1-2 keV band. More recently Gotthelf \& Wang (2000) presented a high resolution Chandra HRC observation of PSR B0540-69.3 which clearly shows emission from the outer shell.

In this letter, we report the results of observations of SNR 0540-69 acquired with the Reflection Grating

Send offprint requests to: K. J. van der Heyden

* Based on observations obtained with XMM-Newton, an ESA science mission with instruments and contributions directly funded by ESA Member States and the USA (NASA).
Spectrometer on board the XMM-Newton Observatory (den Herder et al. 2001). These observations allow us for the first time to detect and identify emission lines from the remnant shell. Our spectral analysis allows us to derive values for the temperature, ionization timescale, abundances, location, and velocity of the emitting gas.

\section{Observations and data reduction}

PSR B0540 was observed repeatedly as part of the calibration of the Reflection Grating Spectrometer (RGS) during the Calibration/Performance Verification (Cal/PV) phase. For the purpose of this letter, we have analyzed the 126 ks data obtained on 2000 May 26, which is the longest observation with the lowest background. The observation was performed with the telescope rolled such that the RGS dispersion axis was aligned at $21.29^{\circ}$, clockwise on the sky, from celestial north. The data were initially processed with the XMM-Newton Science Analysis Software (SAS). The spectra were extracted by applying spatial filters to the CCD image while a CCD pulse-height filter was applied to select the $m=-1$ spectral order. The remnant is located on the edge of a broad region of diffuse emission (Wang \& Helfand 1991) which complicates background determination. However, the image is small enough on the detector that spatial regions offset from the source position can be used for background subtraction. The background spectra were extracted by applying the same pulse height filters as used for the source, to spatial regions at either edge of the camera. 

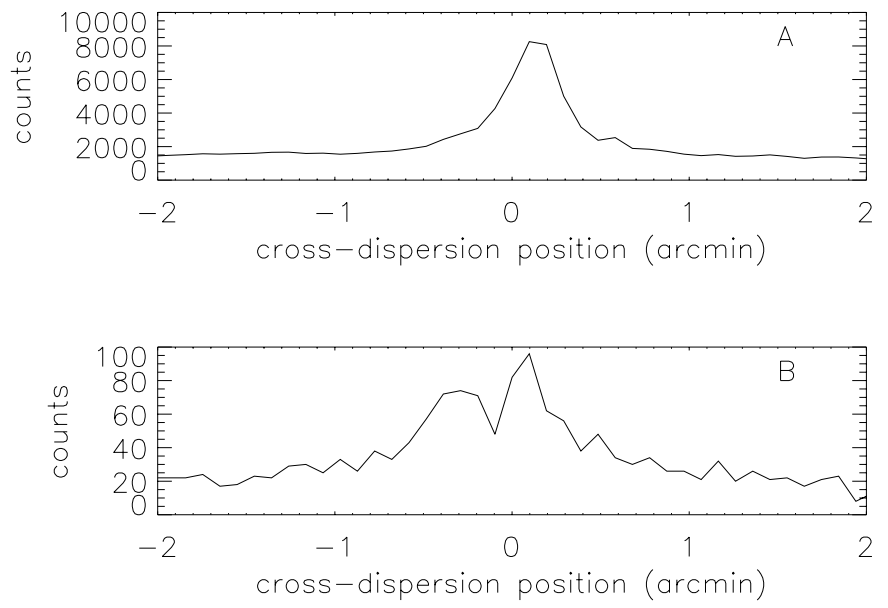

Fig. 1. Cross-dispersion profiles of SNR 0540-69 obtained by integrating along the dispersion axis for a) the entire spectrum and b) O VIII Ly $\alpha$ only

A response matrix appropriate to the spatial extent of the source was generated as follows. A spatial mask corresponding to the RGS aperture was imposed on the Chandra HRC image, and the intensity distribution was integrated over the RGS cross-dispersion direction. The resulting profile was convolved with the RGS point source response matrix, generated with the SAS task RGSRMFGEN. The spectral analysis was performed using the SRON SPEX (Kaastra et al. 1996) package, which contains the MEKAL code (Mewe et al. 1995) for modeling thermal emission.

\section{Analysis and results}

\subsection{Location of emitting region}

We initially extracted spectra with spatial filters $0.25^{\prime}$, $0.5^{\prime}, 1^{\prime}$ and $1.5^{\prime}$ wide, each centered about the peak emission. The most interesting feature in the spectra is the presence of an O VIII Ly $\alpha$ emission line that becomes more prominent as the width of the spatial extraction region is increased. This is an indication that at least some of the O emission is from the SNR shell, and not the central region. To further constrain the spatial distribution of the emitting gas we created a cross-dispersion image profile of the O VIII Ly $\alpha$ line by integrating over the line in the dispersion direction. The cross-dispersion profile, plotted in Fig. 1, shows two peaks. Referring to the Chandra HRC image (Gotthelf \& Wang 2000, reproduced here as Fig. 2), we conclude that we see $\mathrm{O}$ emission both from the central region of the remnant, centered on the pulsar, (the peak at offset zero in Fig. 1b), as well as from the partial shell to the west (the second peak $\approx 24$ arcsec towards more negative offset in Fig. 1b).

The angular extent of this partial shell, $\Delta \phi \approx 30^{\prime \prime}$, corresponds to an effective wavelength width along the dispersion direction of $\approx 0.06 \AA$, comparable to the wavelength resolution of the spectrometer. This implies that we

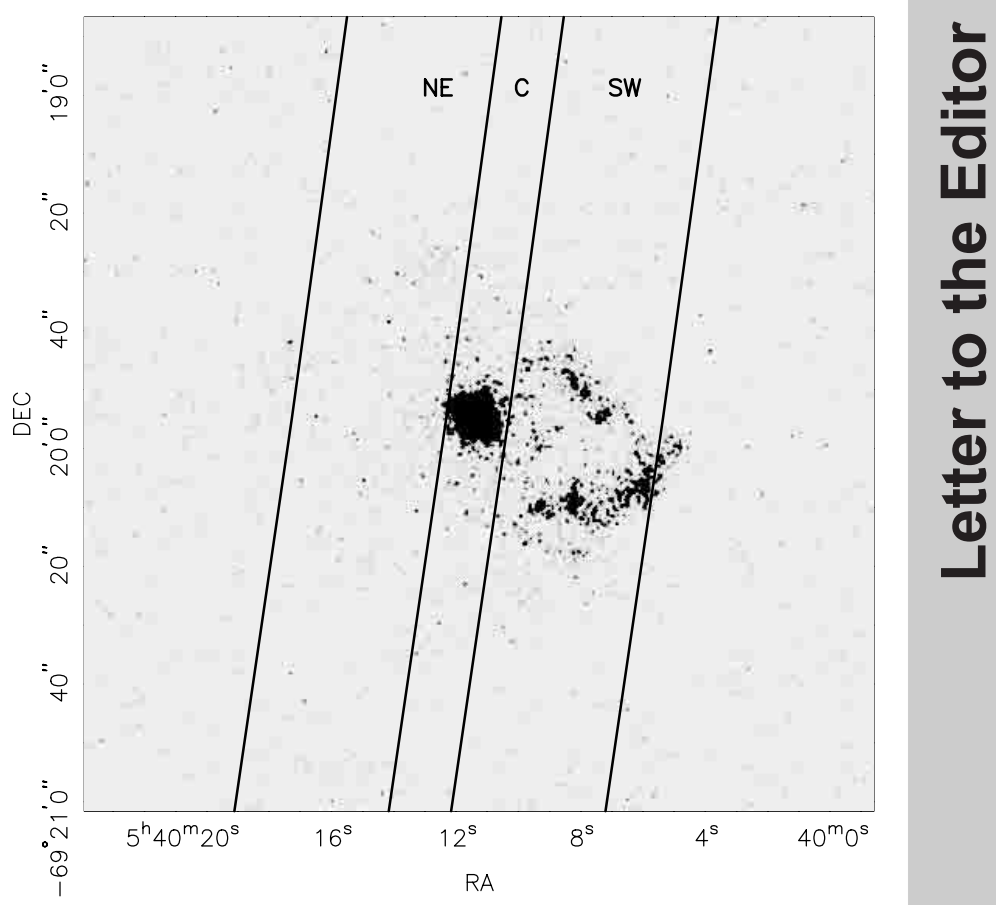

Fig. 2. X-ray image of SNR 0540-69.3 obtained by the Chandra HRC. The image contains the $50 \mathrm{~ms}$ pulsar PSR B0540-69.3, the plerion and the outer shell. The SW, C and NE regions used for spectral analysis are indicated. The disperion direction of the RGS is along the long dimension of the extraction regions

do not have the sensitivity to resolve variations in brightness along the shell.

\subsection{Spectral analysis}

We divided the remnant into three regions, Northeast (NE), Central (C) and Southwest (SW), for the purpose of spectral analysis. For the NE and SW regions we use an extraction width of $0.5^{\prime}$ centered at $\pm 0.375^{\prime}$ about the central compact region, while for the brighter $\mathrm{C}$ region we use a width of $0.25^{\prime}$. The spectra for these regions are displayed in Figs. 3 and 4. Each spectrum shows a strong power law continuum with superimposed line emission, most notably O VIII Ly $\alpha$. Emission line features are weakest in the spectrum extracted from the NE region, where we only detect $\mathrm{O}$ VIII $\mathrm{Ly} \alpha$ and possibly weak O vII emission. The line emission is strongest in the SW spectrum where we easily identify emission lines of O VIII ( $\mathrm{Ly} \alpha, \mathrm{Ly} \beta)$, O VII $(n=2-1$ triplet, $\mathrm{He} \beta)$, Fe XVII $(15.01,16.78,17.05,17.10$ $\AA), \operatorname{Nex}(\operatorname{Ly} \alpha)$, and NeIX ( $n=2-1$ triplet). Due to galactic absorption, no features can be seen longward of $\sim 23 \AA$. Note that even though the shell-like structure and the plerion are only partially resolved by the telescopes of $X M M-N e w t o n$, the high spectral resolution of the RGS allows for high sensitivity to the discrete emission from the remnant, even though the spectrum is dominated by the powerlaw continuum from the plerion.

In the SW spectrum, O viII Ly $\alpha$ has a significant offset of $\Delta \lambda=-0.17 \AA$ with respect to the laboratory wavelength. This offset must be largely due to a Doppler shift, 
and not to a positional offset between the nominal pointing direction (the pulsar position) and the emission centroid of the shell, because the equivalent wavelength extent of the shell is much smaller than the observed shift. In the Chandra image, we find that the centroid of the SW emission, projected onto the RGS dispersion direction, is offset from the centroid of the plerionic emission, by about $\approx-9^{\prime \prime}$ along the dispersion direction. This corresponds to a wavelength offset of $-0.019 \AA$. The net Doppler shift of the SW emission is thus $-0.15 \AA$, which corresponds to a radial velocity of $v \approx-2370 \mathrm{~km} \mathrm{~s}^{-1}$. A similar radial velocity is also observed in the other strong emission lines in the spectrum. Note that the radial velocity of the LMC, $v_{\text {LMC }}=+278 \mathrm{~km} \mathrm{~s}^{-1}$, is only a relatively small correction to this measurement. The emission lines seen in the $\mathrm{C}$ and NE spectra are too faint to permit a meaningful radial velocity determination.

We synthesized a spectral model comprising three components: a power law to represent the synchrotron emission from the plerion, a non-equilibrium ionization (NEI) model for the thermal line emission, and foreground absorption. The free parameters of the model are the photon index of the power law and its normalization, the electron temperature $T_{\mathrm{e}}$, emission measure, abundances, ionization age $n_{\mathrm{e}} t$ of the shocked gas, and the column density $N_{\mathrm{H}}$ of foreground absorbing gas. Here, $n_{\mathrm{e}}$ is the electron density of the shell, and $t$ is the time since the hot gas was shocked. We adopt a distance of $51 \mathrm{kpc}$ throughout.

We concentrated our quantitative analysis on the SW region since the spectrum from this region contains the strongest line emission features. Fixing the $\mathrm{O}$ abundance to a third solar (note that we have no independent way of constraining the absolute abundances, since the continuum emission is dominated by the plerionic emission), the abundances of $\mathrm{Ne}$ and $\mathrm{Fe}$ were allowed to vary with respect to $\mathrm{O}$. An initial fit was made to the SW spectrum, using O VIII Ly $\alpha$ and O viI-triplet only. The parameters obtained were subsequently used as starting parameters for fits to the entire spectrum. For the $\mathrm{Ne} / \mathrm{O}$ and $\mathrm{Fe} / \mathrm{O}$ abundance ratios, we obtain $\mathrm{Ne} / \mathrm{O} \sim 3.0$ and $\mathrm{Fe} / \mathrm{O} \sim 2.1$ relative to their solar values. The best fit model parameters are listed in Table 1. Fits were also made to the NE and $\mathrm{C}$ spectra. Since the line emission in these regions is extremely weak we fixed the NEI component parameters (i.e. electron temperature and ionization parameter) to the best fit parameters obtained from the SW region, while allowing the emission measure and photon indices of the power law component to vary. The best fit parameters are given in Table 1, and the spectra, together with the best fit models are shown in Figs. 3-5.

As can be seen from the Figures and the $\chi^{2}$ values in Table 1 , the fits are not perfect. This is probably due to a combination of remaining small calibration uncertainties in the RGS effective area (most of the $\chi^{2}$ is in the relatively poor fit to the continuum, which is due to the plerion, at the shorter wavelengths, where the RGS effective area is least well calibrated), and the simplicity of our spectral model, which does not allow for temperature and
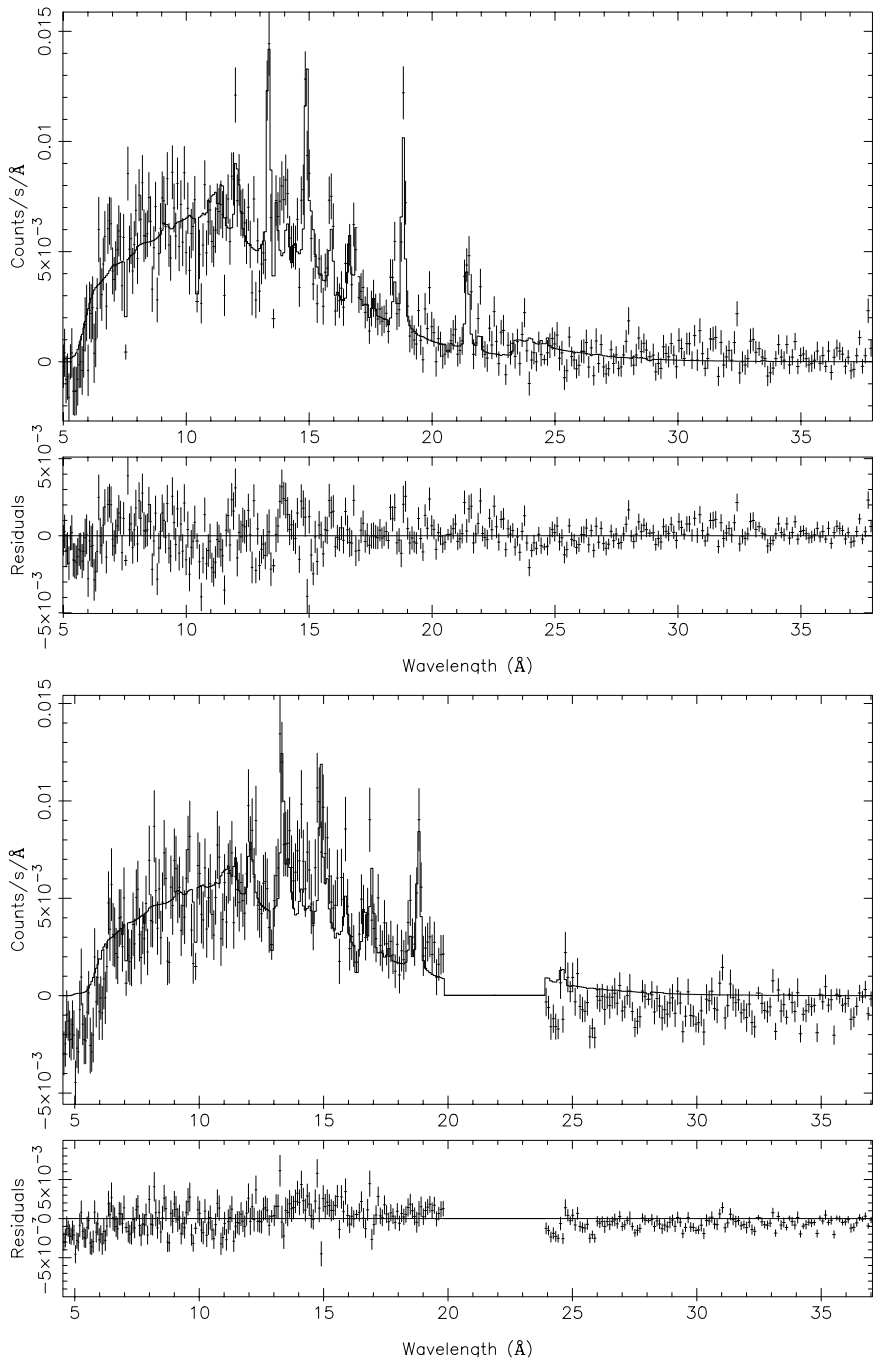

Fig. 3. RGS 1 \& RGS 2 (upper and lower plots respectively) spectrum of the $\mathrm{SW}$ region. The solid line is a model fit to the data. The gap between 20 to $24 \AA$ in the RGS 2 spectra is due to the failed CCD 4 on that instrument. The spectra exhibit a strong power law continuum due to synchrotron radiation from the plerion and thermal line emission from highly ionized atoms of $\mathrm{O}, \mathrm{Ne}$ and $\mathrm{Fe}$

Table 1. Fitting results for the SW, C and NE regions of SNR 0540-69

\begin{tabular}{llll}
\hline parameter & SW & C & NE \\
\hline NEI: & & \\
$n_{\mathrm{e}} n_{\mathrm{H}} V\left(10^{58} \mathrm{~cm}^{-3}\right)$ & $92 \pm 6.5$ & $50 \pm 20$ & $45 \pm 18$ \\
$k T_{\mathrm{e}}(\mathrm{keV})$ & $0.58 \pm 0.18$ & & \\
$n_{\mathrm{e}} t\left(10^{9} \mathrm{~cm}^{-3} \mathrm{~s}\right)$ & $\begin{array}{l}25.22 \pm 5 \\
\end{array}$ & & \\
POW: & & & \\
photon index & $1.82 \pm 0.1$ & $1.2 \pm 0.04$ & $1.75 \pm 0.1$ \\
ABS: & & & \\
$N_{\mathrm{H}}\left(10^{21} \mathrm{~cm}^{2}\right)$ & $3.88 \pm 1$ & & \\
& & 2.8 & 2.9 \\
\hline$\chi^{2} /$ d.o.f. & 2.04 &
\end{tabular}

ionization inhomogeneities. In particular, there is a feature at $\approx 14 \AA$, which is not fit by our isothermal model. Candidate emission lines are $2 \mathrm{p}-3 \mathrm{~d}$ in Fe XVIII at $14.208 \AA$, 

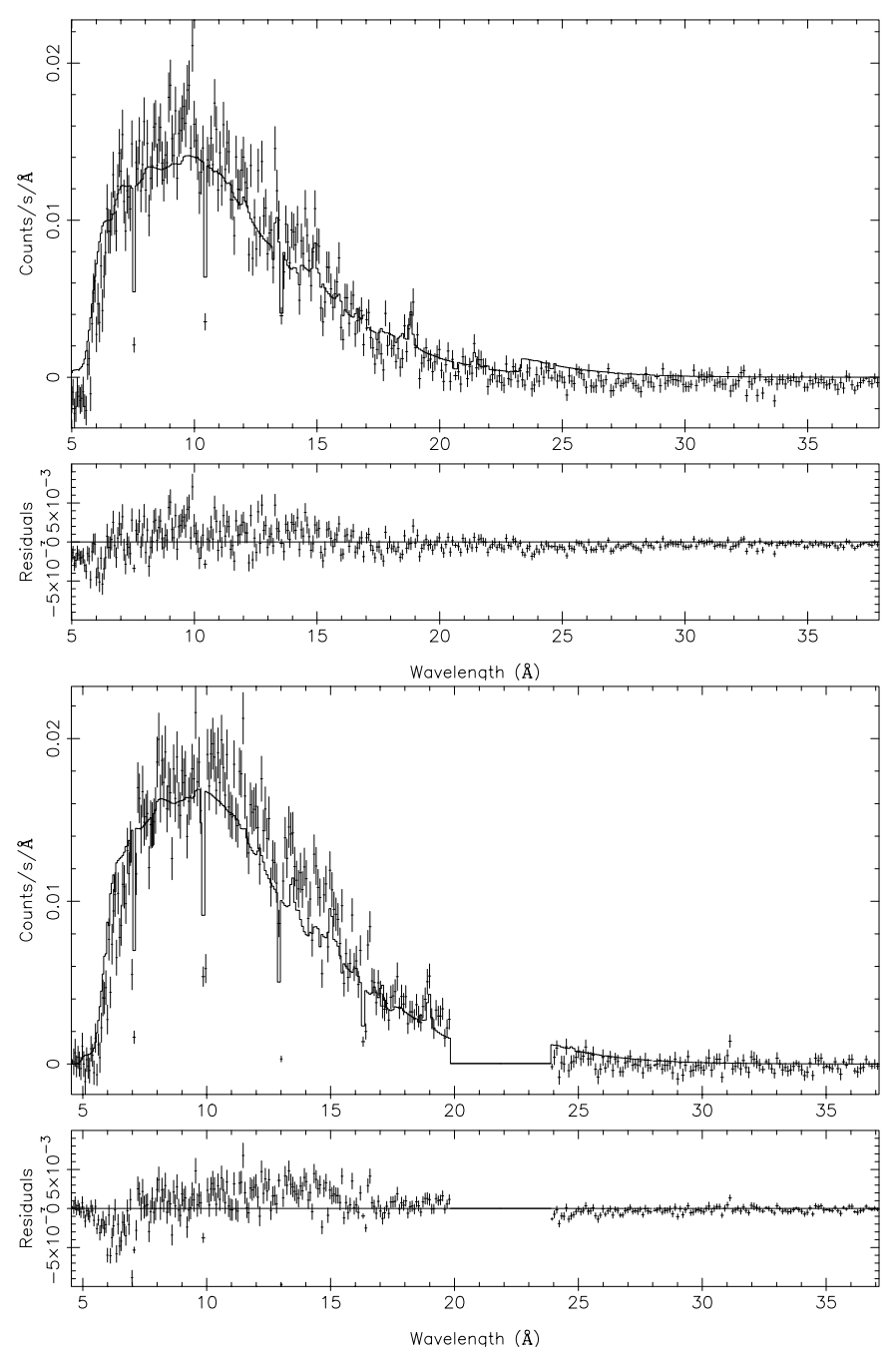

Fig. 4. Same as for Fig. 3, but for the $\mathrm{C}$ region

or possibly $2 \mathrm{~s}-3$ p in Fe XXI at $14.008 \AA$, although the latter should be accompanied by strong emission at $12.29 \AA$ in equilibrium. In any case, detectable amounts of higher charge states of Fe L would definitely indicate the presence of hotter plasma.

\section{Discussion}

First of all, a qualitative point. We detect faint, but significant O VIII emission from the direction of the plerion. At our spatial resolution, we cannot exclude the possibility that a significant component of this line emission is in fact from foreground remnant gas seen in projection and contamination by the SW region. If confirmed at higher spatial resolution, this detection would be interesting, because Mathewson et al. (1980) detected intense optical [O III] emission from this same inner region, with a large velocity dispersion of $v \approx 3000 \mathrm{~km} \mathrm{~s}^{-1}$. There may consequently be a wide range of ionization present in the plerion. Unfortunately, we do not have the sensitivity to try and localize the highly ionized oxygen, and to constrain its kinematics for comparison with the [O III] image.
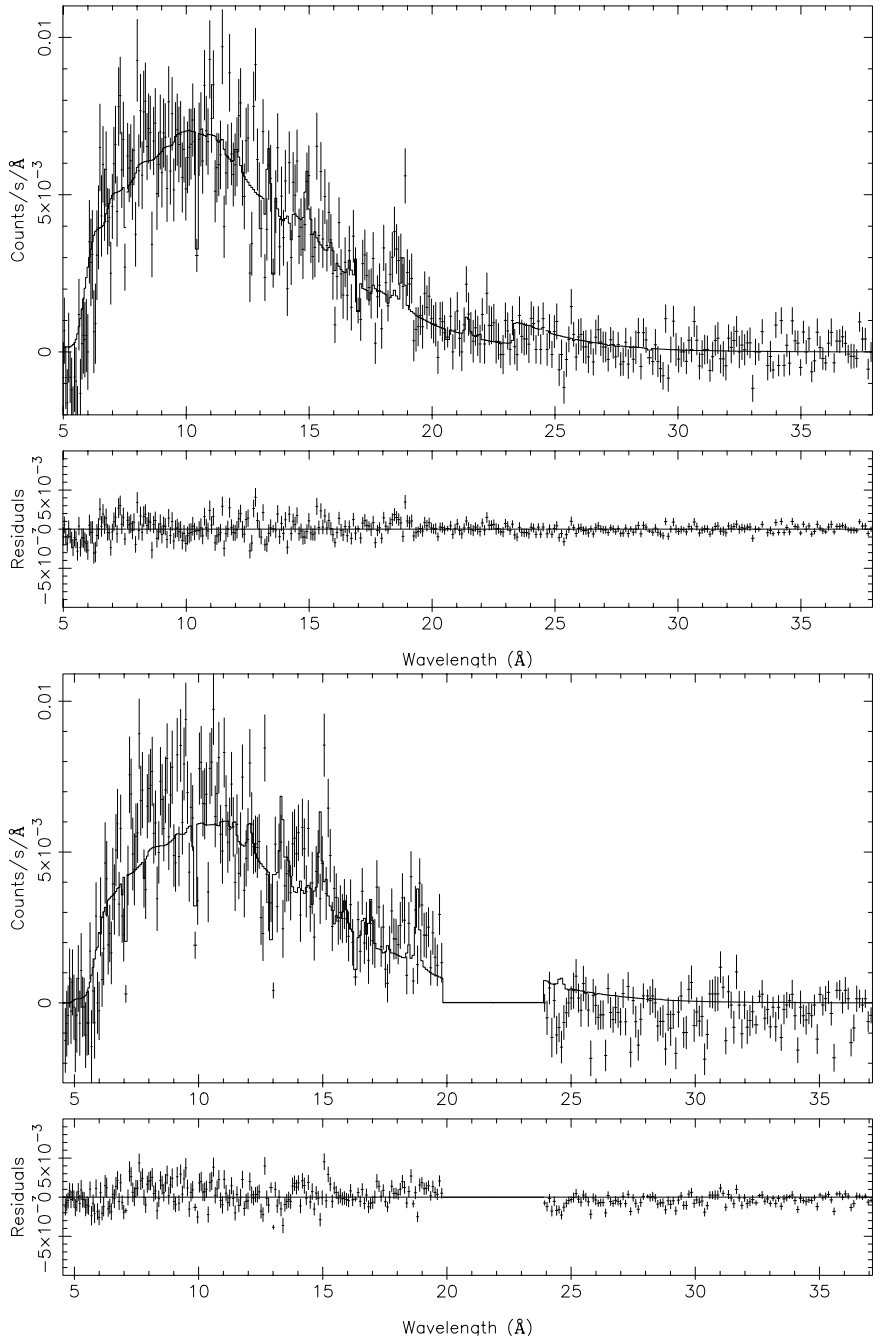

Fig. 5. Same as for Fig. 3, but for the NE region

Next, we check for consistency of our measured parameter values. The interpretation of the radial velocity measured in O VIII is somewhat complicated. We see what appears to be a limb-brightened shell in the Chandra HRC image, but if the emission is really arranged in such a shell, one would not expect to see any radial velocity shift of the material at all, its velocity vector being entirely perpendicular to the line of sight. We know instead that the line emitting material has a velocity component along the line of sight of $\approx-2370 \mathrm{~km} \mathrm{~s}^{-1}$, which, combined with an unknown perpendicular velocity component, sets a lower limit on the true space velocity of the O VIII emitting gas. To relate this space velocity to the velocity of the expanding blast wave requires making a further assumption about the structure and evolution of the expanding remnant. However, the measured radial velocity still gives a lower limit on the true expansion velocity, independent of these assumptions.

With a measured radius of $\approx 30^{\prime \prime}$, the shell has a linear radius of $R_{\mathrm{S}}=7.4 \mathrm{pc}$. Combined with the lower limit on the expansion velocity, we estimate an upper limit to the age of the shell of $t<2800 \mathrm{yr}$, consistent with the 
pulsar spindown age of $1660 \mathrm{yr}$. Conversely, if we assume that the age of the shell is identical to the pulsar spindown age, we would conclude that the true expansion velocity of the blast wave is $\approx 4600 \mathrm{~km} \mathrm{~s}^{-1}$, and the radial velocity we observe in the X-ray lines is either due to material well inside the interior of the remnant (where the flow velocities are smaller), or the line emitting gas must have a substantial perpendicular velocity component if it is located in the immediate post-shock region.

The discrete emission line spectrum implies an ionization age of approximately $n_{\mathrm{e}} t \sim 2.510^{10} \mathrm{~cm}^{-3} \mathrm{~s}$, a low value, which probably implies that the plasma has not yet reached ionization equilibrium. Combined with the pulsar spindown age, one infers a density of the medium of $n_{\mathrm{e}} \sim 0.4 \mathrm{~cm}^{-3}$. But the gas may have been shocked (much) more recently than the characteristic age of the blast wave, depending on it position within the remnant interior, and so we should regard this density estimate as a lower limit. Also, from the emission measure of the line emitting gas, we derive a density of approximately $n_{\mathrm{e}} \sim 10 \mathrm{~cm}^{-3}$ (assuming a half shell of relative thickness $\Delta R \sim R_{\mathrm{s}} / 10$ for the volume estimate). Given the relative insensitivity of the density estimate to the volume and emission measure, this higher density estimate may be closer to the characteristic values at the shell than the estimate derived from the ionization age, and this would favor the conclusion that the gas has been shocked more recently. In fact, the measured electron temperature, $k T_{\mathrm{e}} \sim 0.6 \mathrm{keV}$, is incompatible with the lower limit on the blast wave velocity $v_{\mathrm{s}}>2400 \mathrm{~km} \mathrm{~s}^{-1}$ (unless the electron- and ion temperatures have not equilibrated), and most likely, we are seeing material that has recently passed through a (much slower) reverse shock. If gas with a much higher electron temperature is associated with the outer blast wave, it will be difficult to detect it in the XMM-Newton data. Because the remnant is only partially resolved, both the RGS andEPIC data are dominated by strong emission from the plerion, which reduces the sensitivity to detection of hot thermal gas.

\section{Conclusion}

We have detected resolved X-ray line emission from the supernova remnant shell around PSR B0540-69.3, confirming the nature of the shell-like structure seen in previous experiments as a thermal SNR. Unfortunately, given the relatively low signal-to-noise of the observation, the fact that the object is only partially spatially resolved, and the uncertainties in the interpretation of the estimates of the various observable parameters, we can not uniquely decide what the evolutionary status of the remnant is, or what the relation of the line emitting gas is to the overall structure of the remnant.

Acknowledgements. We thank E. Gotthelf for supplying us with his Chandra HRC image. The Laboratory for Space Research Utrecht is supported financially by NWO, The Netherlands Organization for Scientific Research. The Columbia group is supported by the U.S. National Aeronautics and Space Administration. The Mullard Space Science Laboratory acknowledges financial support form the UK Particle Physics and Astronomy Research Council.

\section{References}

Chanan, G. A., Helfand, D. J., \& Reynolds, S. 1984, ApJ, 287, L23

den Herder, J. W., Brinkman, A. C., Kahn, S. M., et al. 2001, A\&A, 365, L7

Gotthelf, E. V., \& Wang, Q. D. 2000, ApJ, 532, L117

Kaastra, J. S., Mewe, R., \& Nieuwenhuijzen, H. 1996, in UV and X-ray Spectroscopy of Astrophysical and Laboratory Plasmas, ed. K. Yamashita, \& T. Watanabe (Tokyo, Univ. Ac. Press), 411

Mathewson, D. S., Dopita, M. A., Tuohy, I. R., \& Ford, V. L. 1980, ApJ, 242, L73

Mewe, R., Kaastra, J. S., \& Liedahl, D. A. 1995, Legacy, 6, 16 Seward, F. D., Harnden, Jr F. R., \& Helfand, D. J. 1984, ApJ, 287, L19

Seward, F. D, \& Harnden, Jr F. R. 1994, ApJ, 421, 581

Wang, Q. D., \& Helfand, D. J. 1991, ApJ, 379, 327 